\title{
Remodelling of human hamstring autografts after anterior cruciate ligament reconstruction
}

\author{
Rob P. A. Janssen $\cdot$ Jasper van der Wijk • \\ Anja Fiedler • Tanja Schmidt $\cdot$ Harm A. G. M. Sala • \\ Sven U. Scheffler
}

Received: 21 July 2010/ Accepted: 24 January 2011/Published online: 4 February 2011

(C) The Author(s) 2011. This article is published with open access at Springerlink.com

\begin{abstract}
Purpose Histological analysis of the remodelling process of human hamstring tendon (HT) grafts after standardized anterior cruciate ligament reconstruction (ACLR) with an accelerated rehabilitation protocol.

Methods Sixty-seven patients underwent retrieval of midsubstance biopsies after clinically successful hamstring autograft ACLR. Samples were allocated to one of three groups depending on the time point of retrieval: group 1 (6-12 months; $n=15)$, group 2 (13-24 months; $n=16$ ) and group 3 ( $>24$ months; $n=11$ ).

Biopsies from native HT $(n=17)$ and ACL $(n=8)$ served as controls. Cellular density, vascular density and myofibroblast density and collagen fibril alignment were analysed by haematoxylin-eosin, Masson-Goldner-Trichrom and immunohistochemical staining protocols.

Results Compared with native HT $\left(330.4 / \mathrm{mm}^{2}\right)$, total cell number was increased in groups 1-3 (Group $1=482.0$ / $\mathrm{mm}^{2}(P=0.036)$; group $2=850.9 / \mathrm{mm}^{2}(P=0.005)$; and group $3=595.6 / \mathrm{mm}^{2}(P=0.043)$. There were no significant differences between the groups for vessel density. Myofibroblast density was higher in group $2\left(199.6 / \mathrm{mm}^{2}\right)$ compared with native HT $\left(1.9 / \mathrm{mm}^{2}, P=0.014\right)$. Collagen orientation was irregular up to 12 months. Thereafter,
\end{abstract}

R. P. A. Janssen $(\bowtie) \cdot J$. van der Wijk · H. A. G. M. Sala Orthopaedic Center Máxima, Máxima Medical Center, Postbus 90052, 5600 PD Eindhoven, The Netherlands e-mail: r.janssen@mmc.nl

URL: www.rpajanssen.com

\author{
A. Fiedler · T. Schmidt · S. U. Scheffler \\ Department of Orthopaedic Surgery and Traumatology, \\ Center for Musculoskeletal Surgery, Charité, \\ Campus Mitte, University Medicine Berlin, \\ Charité Platz 1, 10117 Berlin, Germany
}

collagen orientation became more regular, adapting to, but not fully restoring, the appearance of the intact ACL. For the first 12 months, cells were predominantly ovoid. Ensuing cell morphology changed to spindle shaped in group 2 and predominantly narrow long cells over 24 months.

Conclusion Human hamstring grafts showed typical stages of graft remodelling, which was not complete up to 2 years after ACLR. The remodelling process in humans was prolonged compared with the results obtained in several animal studies.

Level of evidence Case-control study, Level III.

Keywords Remodelling - Human - Hamstring autograft . Anterior cruciate ligament reconstruction $\cdot$ Myofibroblast

\section{Introduction}

ACL ruptures are a common injury in orthopaedic practice. The ACL is frequently replaced by a tendon autograft or allograft to restore normal knee laxity and to prevent the development of early osteoarthritis induced by persistent abnormal laxity [2]. Reconstruction techniques were improved over the last 10 years, but graft failure is not uncommon: 0.7-10\% [12]. Even though substantial research efforts have been presented on various aspects of ACL reconstruction $[2,3,10,13,15,27,28,32-34]$, little is known about the remodelling process of human ACL grafts.

Current studies evaluating human biopsies pose inherent limits: often sample size was small and only a limited number of time points were evaluated postoperatively [6, $11,18,19,21,35]$. Furthermore, use of different types of grafts limits comparability [6, 11]. Studies in humans describe a prolonged remodelling process compared with animal studies [6, 11, 18, 19, 21, 35]. Most animal studies 
found completion of the remodelling process between 24 weeks and one year. The graft undergoes a transition from its initial biological and biomechanical properties to properties resembling the intact ACL. However, a complete restoration of intact ACL properties has not been found, but an adaptation, which has been termed the ligamentization process $[1,4,8,9,14,17,21-23,25,31]$. Knowledge about the duration and the remodelling process itself in humans can potentially influence and improve outcome and rehabilitation protocols following ACL reconstruction $[11,21]$. Aim of this study was to analyse the remodelling process of human hamstring tendon grafts after standardized ACL reconstruction with accelerated brace-free rehabilitation. The hypothesis of the study is that hamstring tendon grafts undergo a remodelling process that will adapt its histological appearance to the morphology of the intact ACL and that this process is completed by one year.

\section{Materials and methods}

Sixty-seven patients underwent retrieval of biopsies between 6 and 117 months after replacement of the ACL from 2001 up to 2007. The biopsies were carried out during second-look arthroscopies, which were not associated with the initial ACL reconstruction. Inclusion criteria were the following: 1. Previous ACL reconstruction by one senior orthopaedic surgeon (HS) using quadruple hamstring autograft with Bone Mulch Screw fixation in femur and WasherLoc fixation on tibia (Arthrotec, Warsaw, IN, USA); 2. No signs of abnormal laxity on clinical examination nor at examination under anaesthesia at the time of second-look arthroscopy. A knee with normal laxity is defined as a knee without giving way sensation, KT $1000<3 \mathrm{~mm}$ differences on $133 \mathrm{~N}$ side-to-side testing (MEDmetric Co., San Diego, CA, USA) and a negative pivot shift test; 3 . Informed consent to participate in the study. Exclusion criteria were the following: 1. Unwillingness to participate in the study; 2. Previous ACL reconstruction by another orthopaedic surgeon or different method of fixation; 3. Abnormal laxity on clinical examination or found in examination under anaesthesia at the time of second-look arthroscopy. Abnormal laxity is defined as a knee with giving way sensation, KT-1000 $>3 \mathrm{~mm}$ differences on $133 \mathrm{~N}$ side-to-side testing and/or positive pivot shift test; 4 . Cyclops lesion, extension deficit or other reasons related to possible ACL graft problems; 5 . Inadequate tissue sample for histological analysis. The study was approved by the medical ethics board of the Máxima Medical Center Eindhoven-Veldhoven, the Netherlands. Written informed consent was documented in all patients.
The ACL reconstructions were performed by the same senior orthopaedic surgeon (HS). The surgical technique for ACL reconstruction was identical in all patients: a trans-tibial technique with Bone Mulch Screw fixation on the femur and WasherLoc fixation on the tibia (surgical technique by S.M. Howell, MD. Fixation materials by Arthrotec, Warsaw, IN, USA). The graft was a doublestrand semitendinosus and double-strand gracilis tendon. Tension on the hamstring autograft at the time of fixation was $90-100 \mathrm{~N}$, with the knee in full extension.

A standardized accelerated rehabilitation programme started on the first day postsurgery. Patients were allowed full weight bearing as tolerated. Crutches were used for the first 2 weeks. In addition to active flexion and extension exercises, the knee was flexed to 90 degrees by means of continuous passive motion machine (OrthoRehab, Oakville, Ontario, Canada). Closed-chain exercises were prescribed on the first day postsurgery. Full range of motion was allowed. After discharge, physiotherapy was continued 2-3 times a week according to a standardized, brace-free, protocol. Unrestricted closed- and open-chain knee-extension exercises were allowed. Resumption of running in a straight line was allowed at $8-10$ weeks. Unrestricted return to heavy work activities was allowed after 3 months and competitive contact sports after 4-6 months.

\section{Histological examination}

A single tissue sample was collected from one of the four strands of the quadruple hamstring autograft. The synovial layer was cleared from the middle section of the graft bundle. A Shutt mini-tip straight forceps $(2.75 \mathrm{~mm}$ diameter, Linvatec, Fl, USA) were used through the anteromedial portal to take a superficial mid-substance biopsy of the hamstring tendon graft bundle. Size of the biopsies was 2-3 mm.

In order to create a timeline of ACL autograft remodelling belonging to different individuals, samples were allocated to one of the following groups depending on the time point of their retrieval after ACL reconstruction: group $1=6-12$ months, group $2=13-24$ months and group $3=$ greater than 24 months. Two control groups were made: native hamstring tendon (HT) and native ACL. The HT control group biopsies were taken in patients (nonrelated to groups 1-3) at the end of ACL reconstructive surgery. A biopsy was taken from the excess of the hamstring autograft exiting the tibia tunnel after tensioning and fixation of the graft. This excess tendon was normally discarded. The ACL control biopsies were taken from patients (non-related to groups 1-3) who underwent ACL reconstruction after an acute femoral ACL tear (not later than 8 weeks after injury). Therefore, it was possible to obtain tissue samples from non-injured mid-substance 
areas. All patients gave written consent that tissue samples were allowed to be obtained and to be processed histologically. After the biopsies were taken, the remaining tissue was removed to continue with the ACL reconstruction procedure. Directly after retrieval, samples were fixed in formalin for 2 to 3 days, automatically dehydrated for 3 days and embedded in paraffin. Serial cuts $(4 \mu \mathrm{m})$ were prepared and mounted on slides with $3 \%$ silane (Sigma Chemical, St. Louis, MO, USA).

For descriptive and quantitative cell analysis, haematoxylin-eosin (HE) and Masson-Goldner-Trichrom (MG) staining were used following standard histological protocols.

Vascular density was evaluated by immunostaining sections with a polyclonal rabbit anti-human von Willebrandt factor (factor VIII) antibody (cat.-no. M0851, Dako, Glostrup, Denmark). This antibody binds on the endothelial surface of blood vessels.

For the detection of myofibroblasts, tissue sections were immunostained with a mouse anti-human ASMA monoclonal antibody (cat. no. M0851, Dako, Glostrup, Denmark), which binds to $\alpha$-smooth muscle actin $(\dot{\alpha}-$ sma $)$ especially present in myofibroblast.

All tissue samples were hydrated and pre-treated with $0,1 \%$ pronase for $10 \mathrm{~min}$ at $37^{\circ} \mathrm{C}$ for factor-VIII analysis. Myofibroblast detection did not require pre-treatment. For both analyses, $10 \%$ normal horse serum (Vector Laboratories Inc., Burlington, CA, USA) was used for $20 \mathrm{~min}$ at room temperature to block non-specific binding sites. The antibody was diluted 1:200 for factor-VIII and 1:100 for $\dot{\alpha}$-sma and added to the tissue samples overnight in a humidity chamber at $4^{\circ} \mathrm{C}$. After rinsing the samples with tris-buffered saline, they were incubated with the biotinylated horse anti-mouse immunoglobulin G secondary antibody for $30 \mathrm{~min}$. This was followed by incubation with an avidin-biotin complex (ABC Kit, Vectors Laboratories Inc., Burlington, CA, USA) linked with alkaline phosphatase as a reporter enzyme for $50 \mathrm{~min}$. Staining was achieved with Neurofuchsin as a chromogen. Tissues were counterstained with Mayers Haematoxylin, dehydrated and mounted in a xylol-soluble mount (Vitroclud, R Langenbrinck, Emme Both Endingen, Germany).

For all assessments (cellular density, vessel density and myofibroblast density), sections were automatically digitized and saved using a digital video analysis system (KS 400 3.0, Carl Zeiss AG, Göttingen, Germany). Ten regions of interest (ROI) of different sizes, depending on the sample size, were placed on the sample at random. Cells, vessels and myofibroblasts were counted per $\mathrm{mm}^{2}$ at $\times 100$ power. Values are reported with 1 decimal. Myofibroblasts were morphologically differentiated from perizytes. These cells vary by their cell shape, the proximity to vessels and show a different distribution between matrix fibres.
For descriptive analysis, cell distribution pattern (uniform/not uniform), morphology (oblong/spindled/rounded, ovoid) and the evidence of inflammatory reactions were analysed at $\times 50, \times 100, \times 200$ and $\times 400$ power. Collagen fibril alignment was also assessed.

\section{Statistical analysis}

A Shapiro-Wilks test was used to evaluate the normal distribution of all parameters of interest. Because of nonparametric distribution, the Kruskal-Wallis test was performed for group comparison. The Pearsons' chi-square test was used for comparison of gender. Finally, the MannWhitney $U$ test was used to compare cellular density, vascular density and myofibroblast density pairwise between the three groups, native HT and native ACL. Results were corrected with Bonferroni-Holm correction. Level of significance was set at $P \leq 0.05$.

\section{Results}

Total number of biopsies (one per patient) was 67: 15 biopsies in group 1 (6-12 months after ACL reconstruction), 16 in group 2 (13-24 months), 11 in group 3 (>24 months), 17 HT control and 8 ACL control. Groups 1-3 were statistically comparable for age, gender, activity level and results of the KT 1000 measurements (Table 1). Table 2 shows the detailed results for cell, vessel and myofibroblast density for all biopsy groups.

Compared with native HT, total cell number was significantly increased in groups 1-3 (group 1: $P=0.036$; group 2: $P=0.005$; and group 3: $P=0.043$ ). The highest value was found at 13-24 months (Fig. 1). Total cell number decreased from group 2 to group 3 without reaching the cell density level of the native ACL (N.S.).

Figure 2 illustrates the results for vessel density. Vessel density showed the lowest value in group 1. Consecutively, vessel density increased up to the level of native HT in group 2 and higher values in group 3, without reaching the vessel density of the native ACL at any time point (N.S.). Comparing the controls, HT had a lower vessel density than ACL (N.S.).

The results for myofibroblast density are illustrated in Fig. 3. Myofibroblast density was significantly higher in group 2 compared with native HT $(P=0.014)$. Myofibroblast density increased from group 1 to group 2 (N.S.). From group 2 to group 3, myofibroblast density decreased but was still increased compared with both controls (N.S.). Figure 4 demonstrates myofibroblast histology in the 3 study groups.

Necrosis was absent in the biopsies. Collagen orientation was irregular up to 12 months. Thereafter, collagen orientation became more regular, adapting to, but not fully 
Table 1 Demographics (age, gender) of all study groups and KT 1000 results (133 N difference between injured and non-injured leg)

\begin{tabular}{|c|c|c|c|c|c|}
\hline & $\begin{array}{l}\text { Age } \\
\text { Median (range) } \\
\text { Mean (SD) }\end{array}$ & & Gender $(\mathrm{m} / \mathrm{f})$ & & $\begin{array}{l}\text { KT } 1000133 \mathrm{~N} \\
\text { Inj. - Non-inj. } \\
\text { Mean (SD) }\end{array}$ \\
\hline Group 1 & $28.0(19-54)$ & $P=0.461$ & $9 / 6$ & $P=0.932$ & $0.8(0.8)$ \\
\hline$<12$ months $(\mathrm{n}=15)$ & $31.1(11.8)$ & & & & \\
\hline Group 2 & $25.5(17-46)$ & & $10 / 6$ & & $0.6(0.7)$ \\
\hline $13-24$ months $(\mathrm{n}=16)$ & $27.7(8.7)$ & & & & \\
\hline Group 3 & $29.0(21-37)$ & & $7 / 10$ & & $1.0(0.8)$ \\
\hline$>24$ months $(\mathrm{n}=11)$ & $29.0(6.3)$ & & & & \\
\hline
\end{tabular}

Table 2 Results of all biopsy groups for cellular density, vessel density and myofibroblast density

\begin{tabular}{|c|c|c|c|c|c|}
\hline & Group 1 & Group 2 & Group 3 & HT control & ACL control \\
\hline \multicolumn{6}{|l|}{ Cells $/ \mathrm{mm}^{2}$} \\
\hline Mean & 736.3 & 789.5 & 718.2 & 327.6 & 535.8 \\
\hline Range & 1550.7 & 1243.5 & 1173.2 & 663.3 & 1545.9 \\
\hline Median & 482.0 & 850.9 & 595.6 & 330.4 & 371.9 \\
\hline SD & 454.1 & 371.1 & 386.9 & 201.6 & 489.4 \\
\hline \multicolumn{6}{|c|}{ Vessels $/ \mathrm{mm}^{2}$} \\
\hline Mean & 17.9 & 6.1 & 8.2 & 7.4 & 11.9 \\
\hline Range & 181.8 & 41.9 & 22.6 & 67.7 & 31.5 \\
\hline Median & 0.0 & 3.4 & 3.6 & 3.1 & 10.1 \\
\hline SD & 47.3 & 10.3 & 8.5 & 15.9 & 9.3 \\
\hline \multicolumn{6}{|c|}{ Myofibroblast $/ \mathrm{mm}^{2}$} \\
\hline Mean & 35.6 & 224.1 & 207.9 & 3.4 & 5.7 \\
\hline Range & 144.7 & 820.6 & 815.0 & 14.6 & 25.7 \\
\hline Median & 11.5 & 199.6 & 97.7 & 1.9 & 1.4 \\
\hline SD & 47.3 & 234.4 & 275.3 & 4.4 & 8.9 \\
\hline
\end{tabular}

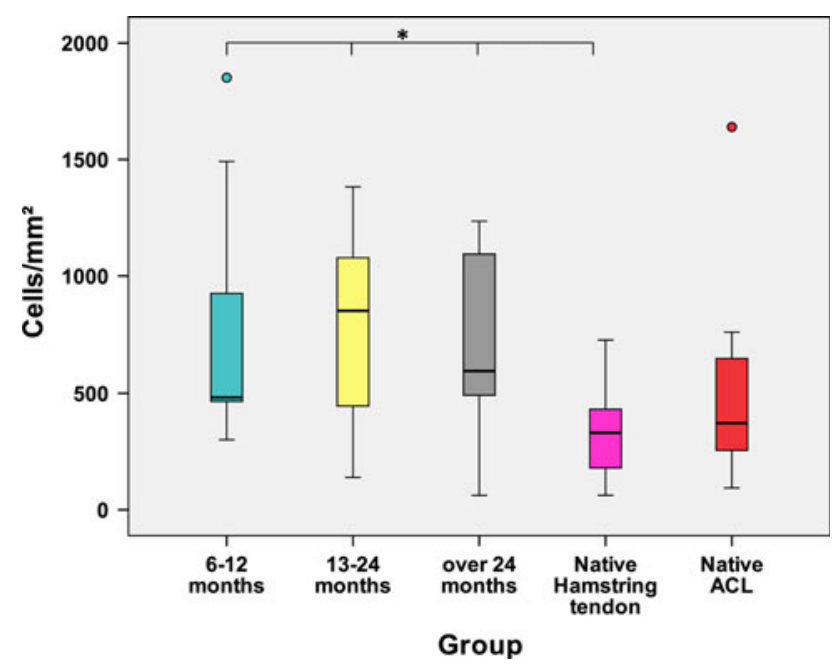

Fig. 1 Results of the median cellular density for all biopsy groups. Significant differences between groups $1-3$ and native hamstring tendon are illustrated with *

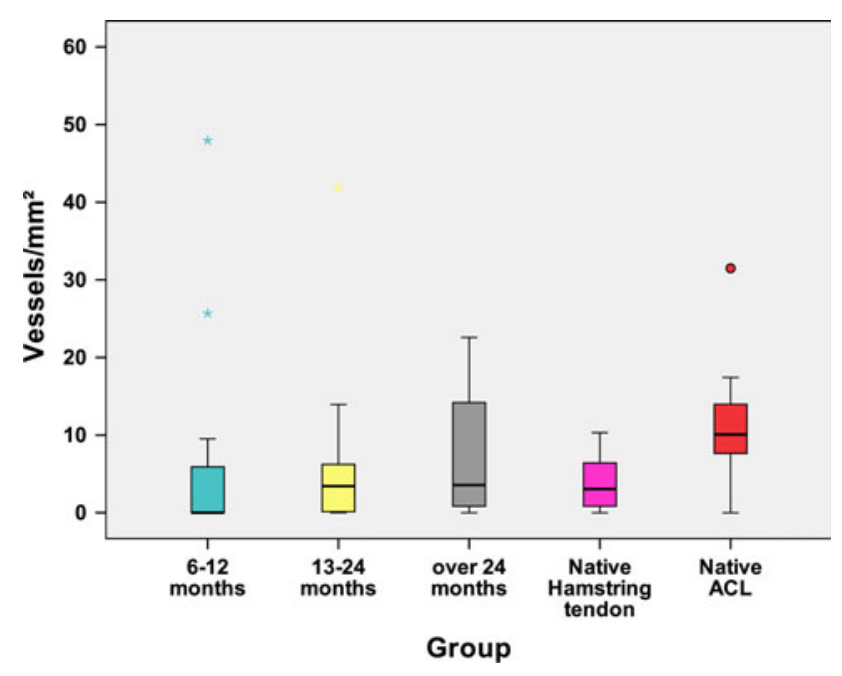

Fig. 2 Results of median vessel density for all biopsy groups

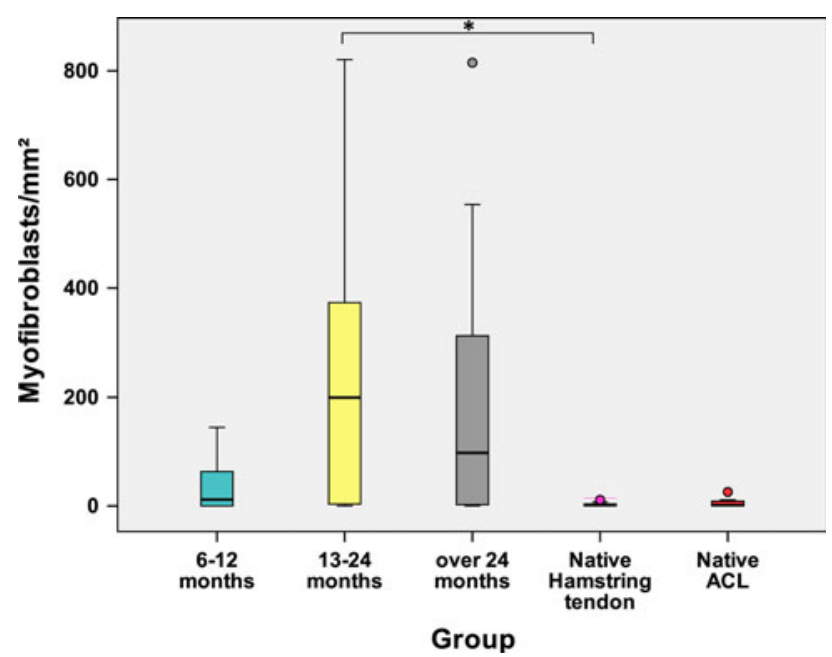

Fig. 3 Results for median myofibroblast density for all biopsy groups. Myofibroblast density was significantly higher in group 2 (13-24 months) compared with native HT control (marked with *) 

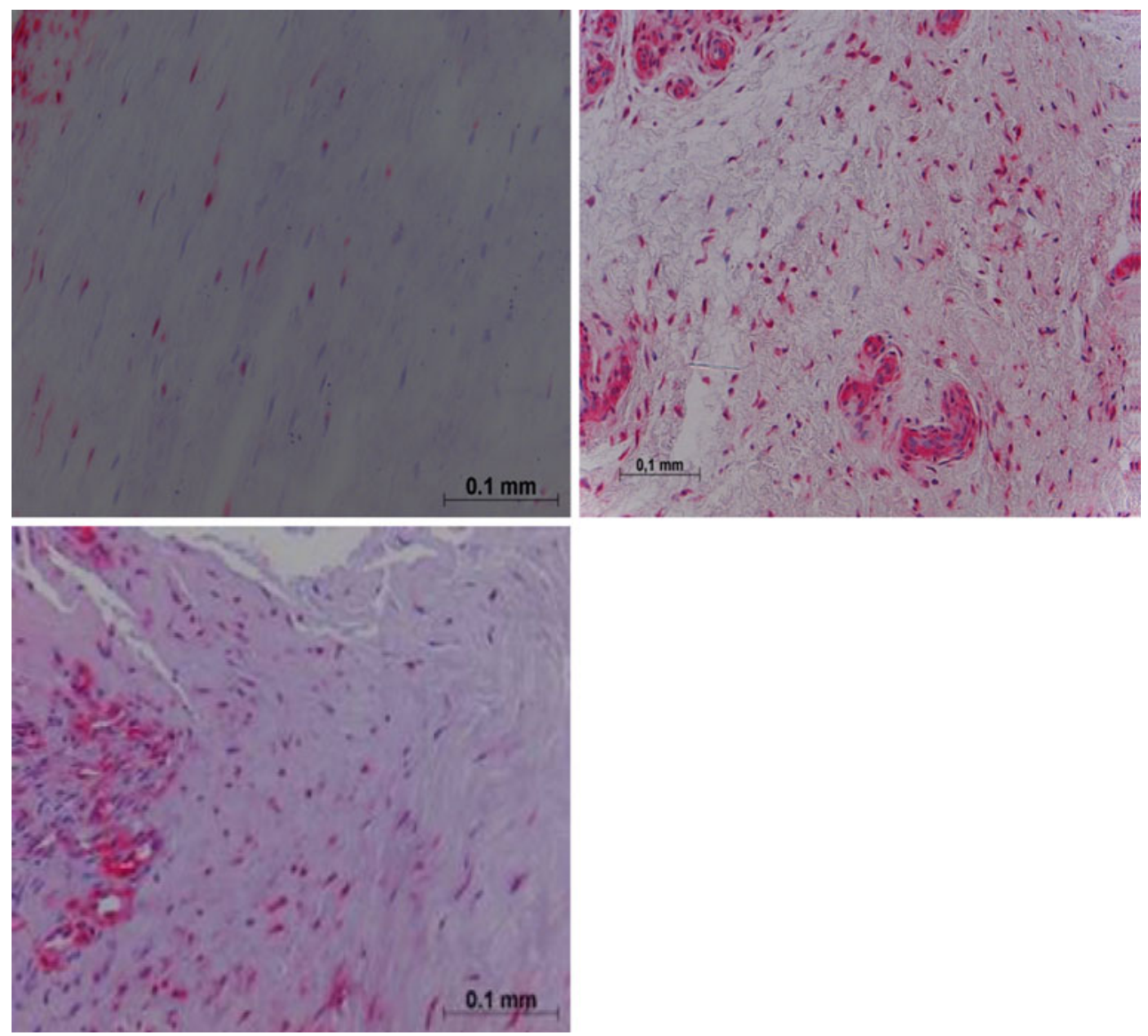

Fig. 4 Alpha-smooth staining: Group 1 (top left) showed a moderate number of myofibroblasts compared with groups 2 and 3 (right and down). Note an increased number of myofibroblast and vessels in groups 2 and 3

restoring, the appearance of the intact ACL. For the first 12 months, cells were predominantly ovoid. Ensuing cell morphology changed to spindle shaped in group 2 and predominantly narrow long cells over 24 months (Fig. 5). Collagen orientation did not return to normal in the study period.

\section{Discussion}

The most important finding of the present study was that human hamstring autografts showed typical stages of graft remodelling, which was not complete up to 2 years after ACL reconstruction.

Animal studies have analysed tendon remodelling in bone tunnels in relation to the type of fixation after ACL reconstruction [7, 16, 24, 29, 30]. Weiler et al. found that biodegradable interference fit fixation of a soft tissue graft may alter the mechanical properties in the early remodelling stage because of tissue compromise at the screw insertion site [30]. Singhatat et al. examined early strength and stiffness of soft tissue fixation comparing biodegradable interference screw versus WasherLoc (Arthrotec, Warsaw,
IN, USA) fixation at 4 weeks. The strength and stiffness of the complex deteriorated after 4 weeks of implantation with the interference screw but was either maintained or improved with the use of the WasherLoc device. They postulated that aggressive rehabilitation after ACL reconstruction should only be allowed after ACL reconstruction with a fixation device that maintains strength and stiffness in the first few weeks of implantation [24]. In the present study, the WasherLoc device was used as tibial fixation of the hamstring tendon autograft. All patients followed a standardized accelerated rehabilitation protocol.

Other animal studies have analysed the intra-articular remodelling process of different graft types after ACL replacement. The autograft underwent necrosis, revascularization and remodelling over time. Return of overall graft integrity and histological appearance was completed 6-12 months after reconstruction without full restoration of the mechanical strength of the intact ACL [4, 8, 25, 26, 31]. Grafts approached only $50-60 \%$ of the intact ACL failure strength [1, 13, 20, 31].

Human biopsy studies have been performed with various techniques to analyse the remodelling of autografts after ACL reconstruction [6, 11, 18, 19, 35]. 

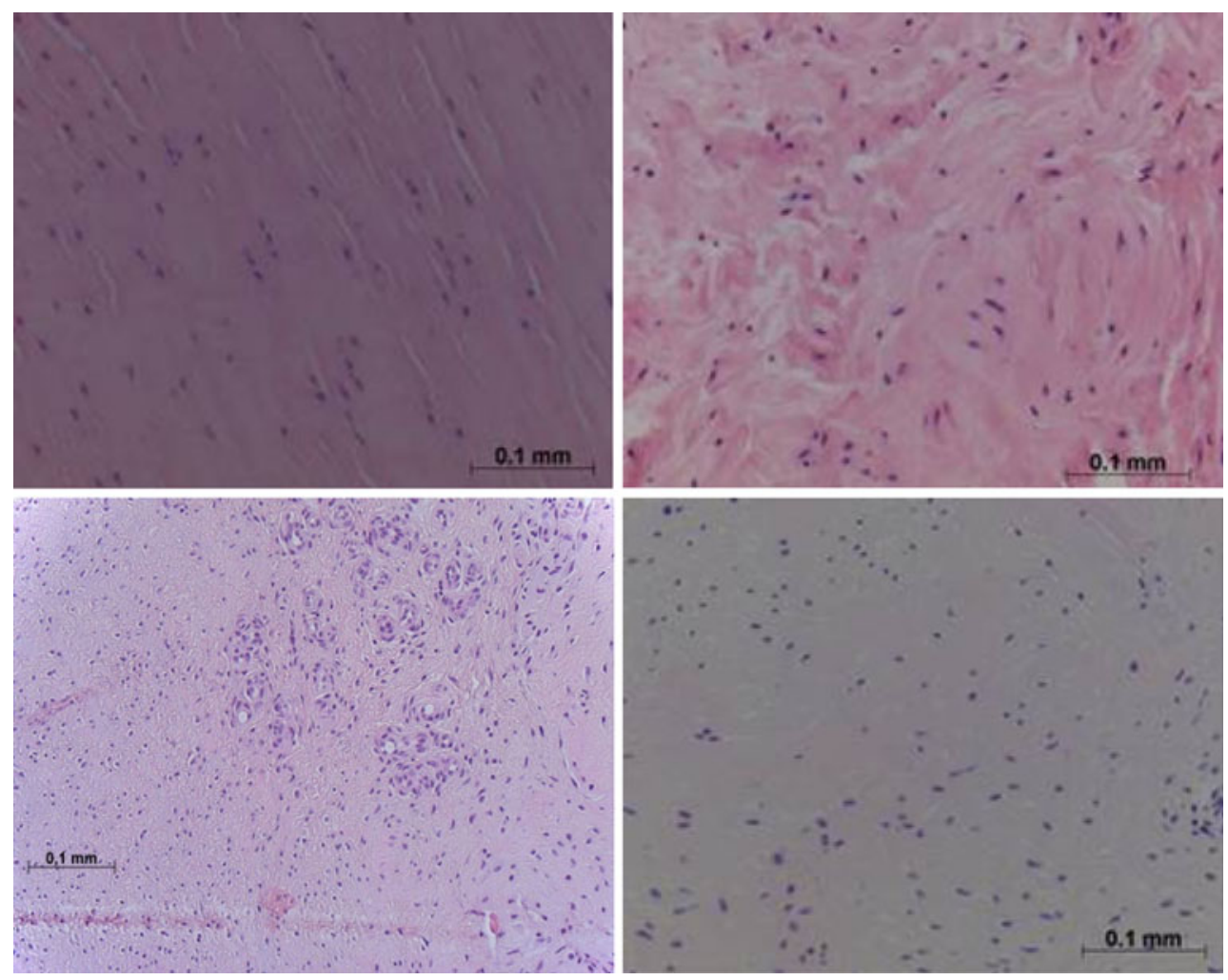

Fig. 5 HE staining: hamstring tendon (top left) with regular collagen orientation, moderate cellular density and little fibroblast activity. Group 1 (top right): irregular collagen alignment, increased cellular

Marumo et al. performed a biochemical study to analyse the remodelling of 30 patellar tendon and 20 hamstring autografts after ACL reconstruction. They analysed collagen content and the amount of reducible and non-reducible crosslinks. Hamstring autograft biopsies were taken at 4- to 6-month and 11- to 13-month intervals. Native ACL, patellar tendon, gracilis and semitendinosus biopsies served as controls (fresh cadavers). They concluded that, based on amount of collagen crosslinks and their architecture, remodelling occurs within one year after ACL reconstruction. The graft did not gain sufficient mechanical functionality. They hypothesized that a low-aggressive rehabilitation programme might seem more desirable, although this was not specifically examined in their study [11]. Their method of tissue biopsy was comparable with the technique of the present study. Both studies examined mid-substance biopsies for the analysis of remodelling of hamstring autografts in clinically successful ACL reconstructions. In contrast to the study by Marumo et al. the present study used a standardized surgical technique with autograft hamstring tendons and a standardized accelerated rehabilitation protocol. Analysis of cellular density, vascular density and myofibroblast density indicated a prolonged remodelling process compared with their study.

Zaffagnini et al. took 10 mid-substance biopsies of patellar tendon autografts from 6 months to 10 years after ACL density, ovoid cell morphology. Group 2. Further increased cellular and vessel density with an irregular collagen orientation (below left). Group 3 (below right): improved regular collagen arrangement

reconstruction. Two patellar tendons and two ACL's served as controls (fresh cadavers). They performed a qualitative and quantitative histological evaluation of collagen fibrils by means of transmission electron microscopy. The patellar tendon autograft underwent a transformation period up to 2 years without reaching the mean diameter and bimodality of a native ACL [35]. In the present study, hamstring tendon autograft biopsies were examined by immunohistochemical evaluation of cellular density, vascular density and myofibroblast density. Despite the differences in methodology, we agree with their findings that human remodelling was prolonged compared with animal studies.

Rougraff et al. took 23 biopsies, $0.75-78$ months after patellar tendon autograft ACL reconstruction. They found four stages of ligamentization, but duration and evolution of ligamentization was different from animal studies. In the first phase (1-2 months), there was a moderate increase in fibroblasts and start of vascular ingrowth. The excessive graft necrosis observed in animal studies could not be confirmed in humans, where necrosis never involved more than $30 \%$ of the graft's biopsies. During the second phase (2-10 months), increase in metabolic active fibroblasts, vessels and irregular collagen fibres occurred. From 1 to 3 years, fibroblast and vessel density decreased. Grafts showed histology similar to a ligament without reaching 
normal ligament cell or vessel density levels. At 3 years, these levels were achieved [18]. In the present study, the earliest biopsy was taken 6 months after ACL reconstruction. This might explain the absence of necrosis since it would be expected in an earlier stage of remodelling [21].

Falconiero et al. analysed 48 biopsies from human patella and hamstring tendons, 3-120 months after ACL replacement. They concluded that remodelling was completed 12 months after ACL reconstruction because no significant differences for vascularity and fibre pattern were evident. They postulated that their results may support early postoperative return to full activity and an accelerated rehabilitation programme [6]. Contrary to the results of Rougraff and Falconiero et al. the present study found an increasing cell and vessel density up to 24 months. Especially the strong increase in myofibroblast density, from 13 up to 24 months, indicated an active remodelling process from 1 to 2 years. Furthermore, vessel density increased over 24 months, whereas cell and myofibroblast density decreased but stayed higher compared with native controls.

Recent work by Sánchez et al. focused on ligamentization of hamstring autografts treated with platelet-rich plasma preparation rich in growth factors (PRGF) after ACL reconstruction. Thirty-seven biopsies were taken 6-24 months after ACL reconstruction. The PRGF-treated grafts showed more remodelling compared with untreated grafts. At 14-18 months, the uniform linear collagen orientation and the spindle-shaped morphology of the native HT differed from the remodelled collagen and the ovoid cells present after ligamentization. Specific information on the duration of the ligamentization process was not described [19]. In the present study, a remodelling process was also evident. In contrast to the aforementioned human biopsy studies, special immunohistochemical staining techniques were performed to detect vessel and myofibroblast density. Unterhauser et al. showed that the factor-VIII staining can improve vessel detection possibility [26]. Myofibroblasts are known to be important cells during the remodelling process of ACL grafts. Their appearance is described as typical for this process $[5,26]$. The increasing myofibroblast density between 13 and 24 months in the present study indicated that the remodelling process in humans was prolonged compared with the results obtained in several animal studies.

In the light of the current insights into ACL surgery, the trans-tibial technique in the present study was a non-anatomic ACL reconstruction. As a result, knee joint motion will not provide the same mechanical stimulus to the healing ACL graft as to the intact ACL [3, 10, 28, 32-34]. Scheffler et al. presented the importance of restoring knee joint mechanics for graft remodelling after ACL reconstruction in order to replicate the loading conditions of the intact ACL [21]. This might explain the prolonged remodelling process in the present study.
There are some limitations to the study. There were no biopsies earlier than 6 months after ACL reconstruction. Therefore, analysis of the early stage of remodelling could not be performed. It was not possible to study ACL remodelling by subsequent hamstring autograft biopsies in the same individuals. Therefore, it was not possible to verify whether changes occurring in grafts from one time point to another may partially be attributed to amongstsubject variation. The present study was limited to small biopsies of the periphery of the ACL graft. It did not provide information on the remodelling process in the graft centre. Finally, the crimp pattern could not be assessed due to the small biopsies compared with animal study models.

Knowledge about the duration of remodelling process of ACL grafts may influence and improve outcome as well as rehabilitation protocols $[11,21]$. This is the first study to document remodelling of human hamstring autografts in relation to a standardized surgical technique and standardized accelerated rehabilitation. The clinical relevance of the study is that, based on the prolonged remodelling of human hamstring grafts, it may be questionable whether an accelerated rehabilitation protocol after non-anatomic ACL reconstruction is to be recommended. Future research should focus on the remodelling process of hamstring grafts in an anatomic ACL reconstruction.

\section{Conclusion}

Human hamstring grafts showed typical stages of graft remodelling, which was not complete up to 2 years after ACL reconstruction. The remodelling process in humans was prolonged compared with the results obtained in several animal studies.

Acknowledgments The authors would like to thank Ineke van Lijnschoten, MD, pathologist at the Máxima Medical Center Eindhoven, the Netherlands, for all her efforts in the early analysis and histological preparation of the biopsies.

Open Access This article is distributed under the terms of the Creative Commons Attribution Noncommercial License which permits any noncommercial use, distribution, and reproduction in any medium, provided the original author(s) and source are credited.

\section{References}

1. Amiel D, Kleiner JB, Roux RD, Harwood FL, Akeson WH (1986) The phenomenon of "ligamentization": anterior cruciate ligament reconstruction with autogenous patellar tendon. J Orthop Res 4:162-172

2. Andersson C, Odensten M, Gillquist J (1991) Knee function after surgical or nonsurgical treatment of acute rupture of the anterior cruciate ligament: a randomized study with a long-term follow-up period. Clin Orthop Related Res 264:255-263 
3. Arnold MP, Kooloos J, van Kampen A (2001) Single-incision technique misses the anatomical femoral anterior cruciate ligament insertion: a cadaver study. Knee Surg Sports Traumatol Arthrosc 9:194-199

4. Clancy WG Jr, Narechania RG, Rosenberg TD, Gmeiner JG, Wisnefske DD, Lange TA (1981) Anterior and posterior cruciate ligament reconstruction in rhesus monkeys. J Bone Joint Surg [Am] 63:1270-1284

5. Dustmann M, Schmidt T, Gangey I, Unterhauser FN, Weiler A, Scheffler SU (2008) The extracellular remodeling of free-softtissue autografts and allografts for reconstruction of the anterior cruciate ligament: a comparison study in a sheep model. Knee Surg Sports Traumatol Arthrosc 16:360-369

6. Falconiero RP, DiStefano VJ, Cook TM (1998) Revascularization and ligamentization of autogenous anterior cruciate ligament grafts in humans. Arthroscopy 14:197-205

7. Goradia VK, Rochat MC, Grana WA, Rohrer MD, Prasad HS (2000) Tendon-to-bone healing of a semitendinosus tendon autograft used for ACL reconstruction in a sheep model. Am J Knee Surg 13:143-151

8. Goradia VK, Rochat MC, Kida M, Grana WA (2000) Natural history of a hamstring tendon autograft used for anterior cruciate ligament reconstruction in a sheep model. Am J Sports Med 28:40-46

9. Gulotta LV, Rodeo SA (2007) Biology of autograft and allograft healing in anterior cruciate ligament reconstruction. Clin Sports Med 26:509-524

10. Kopf S, Forsythe B, Wong AK, Tashman S, Anderst W, Irrgang JJ, Fu FH (2010) Nonanatomic tunnel position in traditional transtibial single-bundle anterior cruciate ligament reconstruction evaluated by three-dimensional computed tomography. J Bone $\mathbf{J}$ Surg [Am] 92:1427-1431

11. Marumo K, Saito M, Yamagishi T, Fujii K (2005) The "ligamentization" process in human anterior cruciate ligament reconstruction with autogenous patellar and hamstring tendons: a biochemical study. Am J Sports Med 33:1166-1173

12. Menetrey J, Duthon VB, Laumonier T, Fritschy D (2008) "Biological failure" of the anterior cruciate ligament graft. Knee Surg Sports Traumatol Arthrosc 16:224-231

13. Ng GY, Oakes BW, Deacon OW, McLean ID, Lampard D (1995) Biomechanics of patellar tendon autograft for reconstruction of the anterior cruciate ligament in the goat: three-year study. J Orthop Res 13:602-608

14. Panni AS, Milano G, Lucania L, Fabbriciani C (1997) Graft healing after anterior cruciate ligament reconstruction in rabbits. Clin Orthop Relat Res 343:203-212

15. Poolman RW, Abouali JAK, Conter HJ, Bhandari M (2007) Overlapping systematic reviews of anterior cruciate ligament reconstruction comparing hamstring autograft with bone-patellar tendon-bone autograft: why are they different? J Bone Joint Surg [Am] 89:1542-1552

16. Rodeo SA, Arnoczky SP, Torzilli PA, Hidaka C, Warren RF (1993) Tendon-healing in a bone tunnel. A biomechanical and histological study in the dog. J Bone Joint Surg [Am] 75:1795-1803

17. Roseti L, Buda R, Cavallo C, Desando G, Fachini A, Grigolo B (2008) Ligament repair: a molecular and immunohistological characterization. J Biomed Mater Res A 84:117-127

18. Rougraff B, Shelbourne KD, Gerth PK, Warner J (1993) Arthroscopic and histologic analysis of human patellar tendon autografts used for anterior cruciate ligament reconstruction. Am J Sports Med 21:277-284

19. Sánchez M, Anitua E, Azofra J, Prado R, Muruzabal F, Andia I (2010) Ligamentization of tendon grafts treated with an endogenous preparation in growth factors: gross morphology and histology. Arthroscopy 26:470-480
20. Scheffler SU, Schmidt T, Gangey I, Dustmann M, Unterhauser FN, Weiler A (2008) Fresh-frozen free-tendon allografts versus autografts in anterior cruciate ligament reconstruction: delayed remodeling and inferior mechanical function during long-term healing in sheep. Arthroscopy 24:448-458

21. Scheffler SU, Unterhauser FN, Weiler A (2008) Graft remodeling and ligamentization after cruciate ligament reconstruction. Knee Surg Sports Traumatol Arthrosc 16:834-842

22. Scranton PE Jr, Lanzer WL, Ferguson MS, Kirkman TR, Pflaster DS (1998) Mechanisms of anterior cruciate ligament neovascularization and ligamentization. Arthroscopy 14:702-716

23. Seitz H, Menth-Ciari WA, Lang S, Nau T (2008) Histological evaluation of the healing potential of the anterior cruciate ligament by means of augmented and non-augmented repair: an in vivo study. Knee Surg Sports Traumatol Arthrosc 16:1087-1093

24. Singhatat W, Lawhorn KW, Howell SM, Hull ML (2002) How four weeks of implantation affect the strength and stiffness of a tendon graft in a bone tunnel: a study of two fixation devices in an extraarticular model in ovine. Am J Sports Med 30:506-513

25. Tohyama H, Yasuda K, Uchida $H$ (2006) Is the increase on type III collagen of the patellar tendon graft after ligament reconstruction really caused by "ligamentization" of the graft? Knee Surg Sports Traumatol Arthrosc 14:1270-1277

26. Unterhauser FN, Bail HJ, Höher J, Haas NP, Weiler A (2003) Endoligamentous revascularization of a free soft tissue graft after ACL reconstruction. Clin Orthop Res Related 414:276-288

27. Vairo GL, Myers JB, Sell TC, Fu FH, Harner CD, Lephart SM (2008) Neuromuscular and biomechanical landing performance subsequent to ipsilateral semitendinosus and gracilis autograft anterior cruciate ligament reconstruction. Knee Surg Sports Traumatol Arthrosc 16:2-14

28. Voos JE, Musahl V, Maak TG, Wickiewicz Pearle AD (2010) Comparison of tunnel positions in single-bundle anterior cruciate ligament reconstructions using computer navigation. Knee Surg Sports Traumatol Arthrosc 18:1282-1289

29. Weiler A, Hoffman RFG, Bail HJ, Rehm O, Sudkamp NP (2002) Tendon healing in a bone tunnel. Part II: histological analysis after biodegradable interference fit fixation in a model of anterior cruciate ligament reconstruction in sheep. Arthroscopy 18:124-135

30. Weiler A, Peine R, Pashmineh-Azar A, Abel C, Sudkamp NP, Hoffman RFG (2002) Tendon healing in a bone tunnel. Part I: biomechanical results after biodegradable interference fit fixation in a model of anterior cruciate ligament reconstruction in sheep. Arthroscopy 18:113-123

31. Xu Y, Ao YF (2009) Histological and biomechanical studies of inter-strand healing in four-strand autograft anterior cruciate ligament reconstruction in a rabbit model. Knee Surg Sports Traumatol Arthrosc 17:770-777

32. Yagi M, Wong EK, Kanamori A, Debski RE, Fu FH, Woo SL (2002) Biomechanical analysis of an anatomic anterior cruciate ligament reconstruction. Am J Sports Med 30:660-666

33. Yamamoto Y, Hsu Y, Woo SL, Van Scyoc AH, Takakura Y, Debski RE (2004) Knee stability and graft function after anterior cruciate ligament reconstruction: a comparison of a lateral and an anatomical femoral placement. Am J Sports Med 32:1825-1832

34. Yoon KH, Bae DK, Cho SM, Park SY, Lee JH (2009) Standard anterior cruciate ligament reconstruction versus isolated singlebundle augmentation with hamstring autograft. Arthroscopy 25:1265-1274

35. Zaffagnini S, De Pasquale V, Marchesini Reggiani L, Russo A, Agati P, Bacchelli B, Marcacci M (2006) Neoligamentization process of BTPB used for ACL graft: histological evaluation from 6 months to 10 years. Knee 14:87-93 